\title{
Direct and Selective Synthesis of a Wide Range of Carbon Nanomaterials by CVD at CMOS Compatible Temperatures
}

\author{
Irene Taurino**1, Arnaud Magrez ${ }^{2}$, László Forró ${ }^{3}$, Giovanni De Micheli ${ }^{1}$, Member, IEEE, \\ and Sandro Carrara ${ }^{1}$, Member, IEEE
}

\begin{abstract}
Biosensors benefit from specific nanostructuration of the active bio-interface layer. In this perspective, a wide range of carbon nanomaterials including multi-walled carbon nanotubes (MWCNTs), nanographite and carbon nanowalls (CNWs) have been directly synthesised by chemical vapor deposition (CVD) on Pt microelectrodes for the first time down to CMOS-compatible temperatures. This integration process, extremely useful to develop nanostructured multi-sensing site biodevices, has been validated by testing sensors for glucose with enhanced and competitive performance. Moreover it paves the way to the full integration of CMOS circuits, nanostructures and bioprobes.
\end{abstract}

Keywords-multi-site biosensors, carbon nanomaterials, CVD, CMOS-compatible temperatures

\section{INTRODUCTION}

$\mathbf{F}$ AST and simultaneous monitoring of many metabolites is an urgent need in fields as medicine, environment and food analysis. In particular, the demand for a rapid and accurate multi-sensing technology is essential for clinicians. The availability of devices that provide the exact concentration of a set of metabolites in realtime favours faster and more appropriate both therapeutic or diagnostic interventions. Theoretically, instrumentation designed for a timely multi-sensing should be able to do several measurements from a small volume and undiluted sample. Consequently, the development of a tiny device is a crucial requirement.

Compared with the historically used analytical techniques (e. g. chromatography, spectrophotometry), the electrochemical detection offers may advantages including high sensitivities, simple use, in situ and continuous monitoring, low cost. The possibility to integrate electrochemical sensors onto simple and portable instrumentation is due to their easy miniaturisation. Interestingly sensors based on miniaturised electrochemical cells have shown remarkable sensitivity and reproducibility when applied

\footnotetext{
1 Laboratory of Integrated Systems, EPFL, Lausanne, Switzerland

2 Crystal Growth Facility, EPFL, Lausanne, Switzerland

${ }^{3}$ Laboratory of Physics of Complex Matter, EPFL, Lausanne, Switzerland

** Contacting Author: EPFL IC ISIM LSI1 INF 334 (Btiment INF) Station 14 CH-1015 Lausanne E-mail: irene.taurino@epfl.ch; Fax: +41 216934 225; Tel: +41216938168
}

to monitor human metabolites [1], [2]. Moreover, small electrodes allow us to design many sensing sites, each one specific for one metabolite, onto a tiny platform creating a multipanel device. Such a kind of sensor is very useful for personalised medicine [3] and possible subcutaneous implantations [4], [5]. The compatibility of their fabrication with the low-cost CMOS technology is extremely useful to implement a full parallel process.

To further improve detection performance, carbon nanomaterials are considered due to their high electrocatalytic activity, large surface area and good support for enzyme immobilisation [6]. Many efforts have been made to integrate carbon nanomaterials onto CMOS-sensors [7], [8], [9]. In particular, metal microelectrodes are historically modified with carbon nanostructures by time-consuming, expensive and hardly-reproducible techniques. Additives (e.g. polymers [10]), commonly employed to help the nanostructuration step, inevitably mask the nanomaterials promising properties and compromise the time-stability of the device due to the binder-matrix instability in aqueous environments. A realistic approach to enable a close coupling of only nanomaterials and electrodes is the direct CVD growth on CMOS wafers. Advantages of the CVD technology are (i) the no post-growth processing (ii) the easy scalability to wafer size and (iii) the wellestablished presence in the semiconductor industry. In our previous work, we have already succeeded in the not trivial growth of carbon nanomaterials on metal microelectrodes [11]. However, the process was performed at high growth temperatures $\left(600-750{ }^{\circ} \mathrm{C}\right)$ that are not compatible with conventional CMOS processes. These temperatures cause irreversible material stresses that inevitably compromise the normal operation of the device with on-board electronic components. To make the whole process compatible with the electronics, the upper limit growth temperature is $450{ }^{\circ} \mathrm{C}$ [12]. Recently, many efforts have been made to lower the synthesis temperatures for fabricating graphitic nanomaterials. However, in the majority of cases, nanomaterials are grown on insulating substrates, on which the effectiveness of the catalyst is more pronounced. Nessim et. al. have already reported the CNT synthesis on metals at CMOS compatible temperatures by thermal decomposition of the hydrocarbon/hydrogen gas mixture. The nanofabrication consisted of only CNTs and was carried out on $\mathrm{Pd}$ and $\mathrm{Ta}$ in a non-selective way. Indeed, in biosensing, 
we need to address specific sites of a platform commonly made of $\mathrm{Au}$ and $\mathrm{Pt}$ [13].

In the present study, we describe a versatile process to integrate a wide range of carbon nanomaterials onto an array of Pt working electrodes of a biosensor by a CVD process down to $450{ }^{\circ} \mathrm{C}$. We successfully satisfied the three major requirements for an effective integration of nanocarbon into microelectrochemical biosystems: 1 . Selectivity with respect to one working electrode (WE) made of $\mathrm{Pt}$ of a multi-sensing platform 2. intimate coupling nanomaterials-electrodes avoiding the use of additional materials. 3. synthesis at CMOS compatible temperatures opening the possibility of a direct integration nanostructures/front-end CMOS data acquisition circuits. Finally, glucose detection, performed by incorporating the glucose oxidase (GODx) as probe-enzyme onto the nanostructured microelectrodes, has shown excellent sensing parameters proving the efficiency of the proposed nanointegration approach in biosensing applications.

\section{MATERIALS AND MethodS}

\section{A. Catalyst electrodeposition and nanocarbon synthesis}

$\mathrm{Fe}_{2}$ Co nanoparticles (NPs) were deposited by a voltammetric method, while chronoamperometry was used to deposit compact $\mathrm{Fe}_{2} \mathrm{Co}$ layers from $\mathrm{Fe}$ and $\mathrm{Co}$ sulphate solutions.

After the catalyst electrodeposition, the devices were firstly placed in the furnace already at the growth temperature and kept there for 10 minutes under $\mathrm{H}_{2}$ and Ar flow $(60 \mathrm{l} / \mathrm{h})$. Then, Ar was introduced at $45 \mathrm{l} / \mathrm{h}$ together with $\mathrm{C}_{2} \mathrm{H}_{2}$ and $\mathrm{CO}_{2}$ (ratio: 1:1; flow: $0.25 \mathrm{l} / \mathrm{h}$ ) for 5 minutes at a temperature ranging between $600{ }^{\circ} \mathrm{C}$ and $450{ }^{\circ} \mathrm{C}$. Then, the chamber was cleaned under Ar flow $(60 \mathrm{l} / \mathrm{h}$ ) for 10 minutes [11]. Before the measurements, a chemical activation (6 hours in sulphuric acid $6 \mathrm{M}$ ) was carried out to increase the electron transfer from the nanostructures [14] and to make the surface more hydrophilic for an easier incorporation of enzymes [15].

\section{B. Chemicals and apparatus}

Cobalt(II) and iron(II) sulfate heptahydrate were purchased from AppliChem. All metal solutions contained 0.5 $\mathrm{M}$ boric acid (AppliChem) and $0.5 \mathrm{M}$ sodium chloride (Sigma). Sulphuric acid from Merck was used for the nanomaterial activation. The glucose detection was carried out by casting $1 \mu \mathrm{l}$ of glucose oxidase $(25 \mathrm{mg} / \mathrm{ml}$, Roche in $2.5 \%$ glutaraldehyde) onto a nanostructured microelectrode and kept overnight before any measurement. The concentration of D-glucose (Sigma) was increased by steps of 100 $\mu \mathrm{M}$ up to $500 \mu \mathrm{M}$ and of $250 \mu \mathrm{M}$ up to $4.5 \mathrm{mM}$. Phosphate buffer saline (10 mM PBS, pH 7.4, Sigma) was employed for the amperometric measurements (applied potential: + $650 \mathrm{mV}$ ) by using an Autolab potentiostat controlled by Nova software. Enzyme-electrodes were stored at $4{ }^{\circ} \mathrm{C}$ under dry conditions. Scanning electron microscope (SEM) (a)

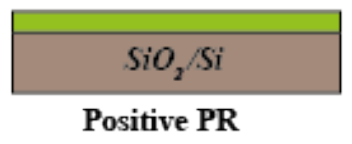

(b)

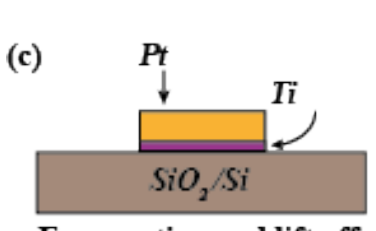

Evaporation and lift off
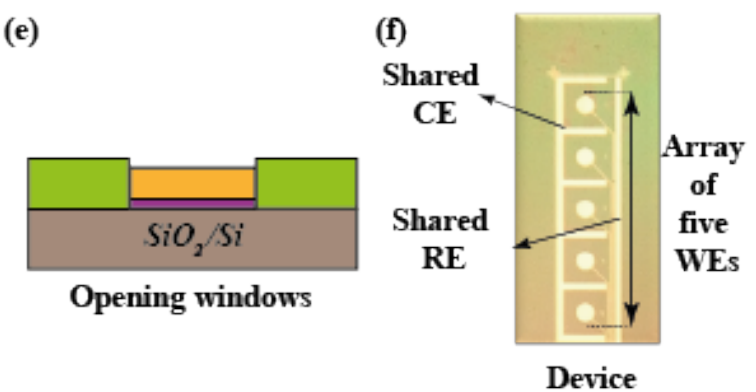

Fig. 1. Schematic of the device microfabrication.

images were recorded with a Zeiss MERLIN SEM. The MWCNT diameter was calculated with an ImageJ software [16]. Filtering and analysis of the data were realised using Igor Pro software (Wavemetrics, Lake Oswego, OR, USA).

\section{RESULTS AND DISCUSSION}

\section{A. Multi-sensing platform fabrication}

The main steps of the device fabrication are shown in Fig. 1. Briefly, we used positive photoresist on $\mathrm{Si}$ wafers with $500 \mathrm{~nm}$ of native $\mathrm{SiO}_{2}$ (Fig. 1 (a)). Platinum (200 $\mathrm{nm}$ ) was deposited by evaporation (Alcatel EVA 600). A buffer layer of $\mathrm{Ti}(20 \mathrm{~nm})$ was added to improve the adhesion between $\mathrm{Pt}$ and $\mathrm{SiO}_{2}$ (Fig. 1 (b) and (c)). After the lift-off, $20 \mathrm{~nm}$ of $\mathrm{HfO}_{2}$ were deposited via atomic layer deposition (BENEQ TFS200) (Fig. 1 (d)). Afterwards, microelectrodes and contacts were introduced on the dielectric material. $\mathrm{HfO}_{2}$ was selectively removed by dry etching (Alcatel AMS 200 DSE) (Fig. 1 (e)). Then, a wafer dicing step produced single devices each one with five working electrodes (diameter of $564 \mu \mathrm{m}$ ). All the working electrodes share the same counter and reference electrode (Fig. 1 (f)). $\mathrm{HfO}_{2}$ was selected as passivation layer for its excellent adhesion to the bottom layers at the growth temperatures. CNTs tend to spontaneously grow onto insulating materials without any predeposited catalyst. To avoid not selective growths, the synthesis time was reduced till no CNTs were seen onto the surface of $\mathrm{HfO}_{2}$.

\section{B. Catalyst electrodeposition}

Electrodeposition was employed as novel, low-cost, versatile and selective technique, even compatible with 

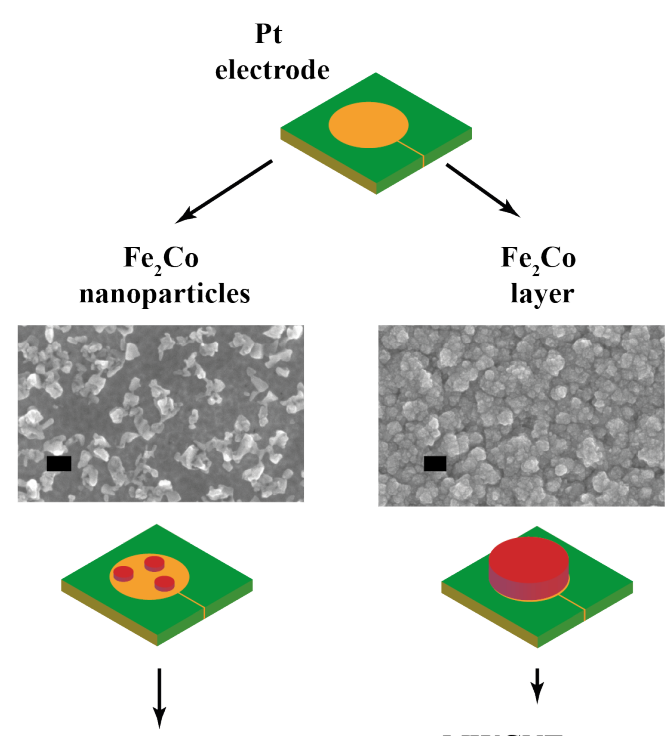

MWCNTs
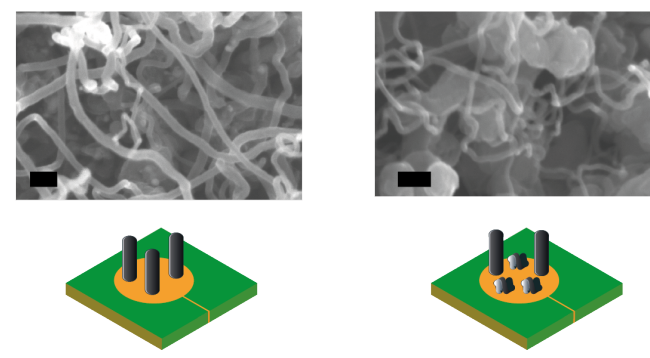

Fig. 2. Schematic of the carbon nanomaterial CVD growth from catalyst nanoparticles (on the left) and from catalyst compact layers (on the right). Black bars: $100 \mathrm{~nm}$.

the platform, to easily obtain different catalyst coatings. Carbon nanomaterials have been previously synthesised from $\mathrm{Fe}$ predeposited onto $\mathrm{Pt}$ electrodes at temperatures ranging from $600{ }^{\circ} \mathrm{C}$ to $750{ }^{\circ} \mathrm{C}$. Below $600{ }^{\circ} \mathrm{C}$, Fe on $\mathrm{Pt}$ was catalytically inactive. The maximum nanocarbon yield for the CVD system we utilised has been previously obtained for $\mathrm{Fe} / \mathrm{Co}$ alloy in a molar ratio 2:1 [17]. The electrodeposition of $\mathrm{Fe}_{2} \mathrm{Co}$ was optimised for both catalyst nanoparticles and coatings. $\mathrm{Fe}_{2} \mathrm{Co}$ nanoparticles resulted form solutions with equimolar concentrations of the two metals (average molar ratio: $2.15 \pm 0.11$ ). On the other hand, $\mathrm{Fe}_{2} \mathrm{Co}$ coatings have been obtained by from sulphate solutions of iron and cobalt in a molar ratio 2:1 (average molar ratio from coatings with different thicknesses is 2.12 $\pm 0.05)$. The morphology of $\mathrm{Fe}_{2} \mathrm{Co}$ nanoparticles and layers is shown in Fig. 2. Fig. 3 clearly shows the increase of the nanocarbon yield when the alloy is used as catalyst material (carbon nanomaterials grown on $\mathrm{Fe}$ (a) and on $\mathrm{Fe}_{2} \mathrm{Co}$ (b) nanoparticles).

\section{Growths down to CMOS compatible temperatures}

a) Synthesis from catalyst nanoparticles: We obtained sparse rolls of MWCNTs (Fig. 2 and Fig. 4 (a))
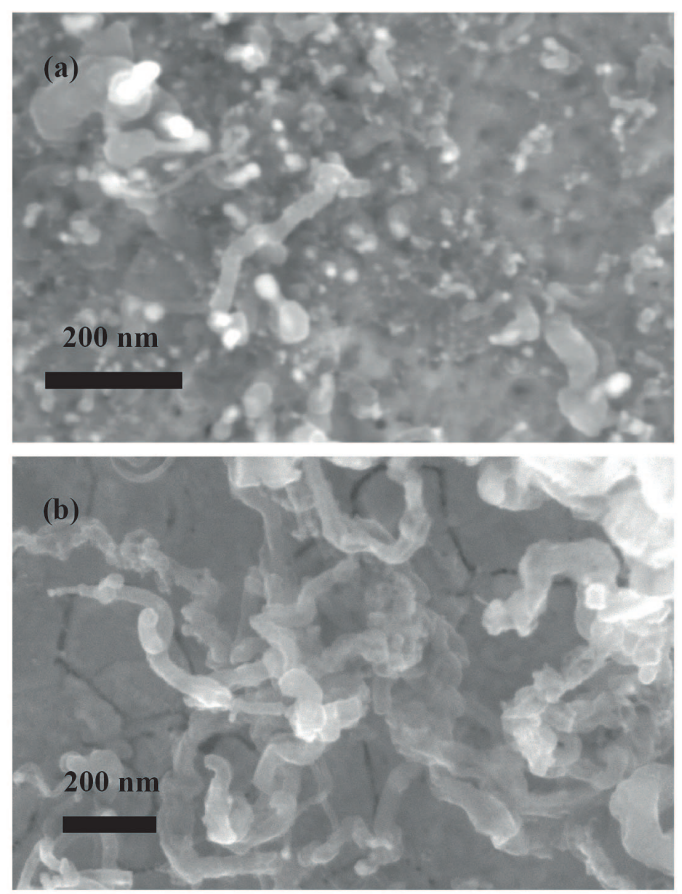

Fig. 3. Increase of the nanocarbon yield passing from $\mathrm{Fe}$ (a) to $\mathrm{Fe}_{2} \mathrm{Co}$ (b) as catalyst.

with an average diameter equal to $(34.4 \pm 19.4) \mathrm{nm}$ using $\mathrm{Fe}_{2} \mathrm{Co}$ nanoparticles. Longer, denser and larger MWCNTs (average diameter: $(52.7 \pm 22.6) \mathrm{nm}$ ) with intercalated nanographite were observed by triplicating the deposition time (from 5 to 15 minutes; Fig. 4 (b) and (c)). Fig. 5 shows that a narrow diameter distribution results from a lower synthesis temperature. In addition, the average diameter decreases going down with the temperature (from $(34.4 \pm 19.4) \mathrm{nm}$ at $600{ }^{\circ} \mathrm{C}$ to $(26.5 \pm 6.5) \mathrm{nm}$ at 450 $\left.{ }^{\circ} \mathrm{C}\right)$. No nanocarbon deposits were obtained at temperature lower than $450{ }^{\circ} \mathrm{C}$.

b) Synthesis from catalyst coatings: Fig. 6 shows the nanocarbon deposits resulting from catalyst coatings with increased thickness. Larger MWCNTs (Fig. 6 (a)) were obtained passing form $2 \mathrm{~s}$ to $4 \mathrm{~s}$ of deposited catalyst. Nanofibers were grown from thinker layers ( $8 \mathrm{~s}$ of catalyst deposition). From $15 \mathrm{~s}$ of electrodeposited $\mathrm{Fe}_{2} \mathrm{Co}$, hybrid nanographite/MWCNTs were produced (Fig. 2 and Fig. 6 (b)). Only nanographite was synthesised from even thicker catalyst coatings (Fig. 6 (c)). The average MWCNT/CNF diameter versus the deposition time is shown in Fig. 6 (d). Once nanographite starts to grow the MWCNT diameter drastically decreases.

We carried out different depositions at temperatures lower than $600{ }^{\circ} \mathrm{C}$ from $\mathrm{Fe}_{2} \mathrm{Co}$ coatings. Nanographite was obtained from thinner $\mathrm{Fe}_{2} \mathrm{Co}$ coatings than using higher temperatures (Fig. 7 (a) and (b)). Also at lower 

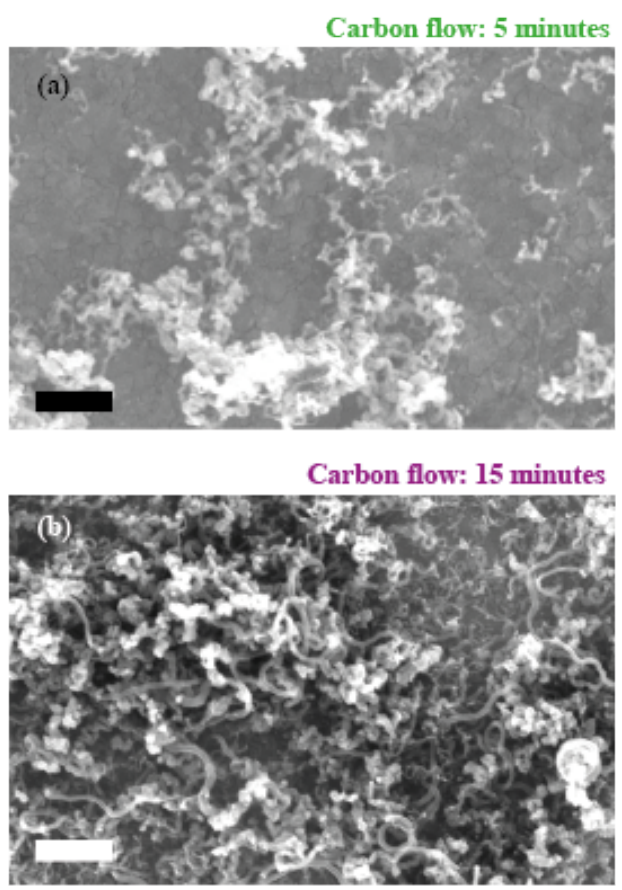

(c)

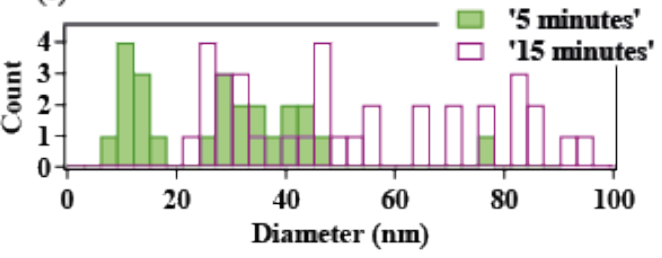

Fig. 4. SEM images of carbon nanomaterials fabricated on catalyst nanoparticles at $600{ }^{\circ} \mathrm{C}$ under 5 (a) and 15 (b) minutes of carbon flow. Bars: $1 \mu \mathrm{m}$. (c) Diameter distribution of MWCNTs produced under two different growth times: 5 (green bars) and 15 (purple bars) minutes.

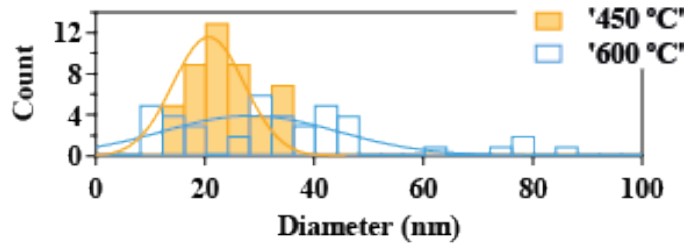

Fig. 5. Diameter distribution of MWCNTs grown from $\mathrm{Fe}_{2}$ Co nanoparticles at $600{ }^{\circ} \mathrm{C}$ (blue) and at $450{ }^{\circ} \mathrm{C}$ (yellow).

temperatures, the synthesis of nanographite determines a simultaneous reduction of the CNT diameter (Fig. 7 (c)). Thicker catalyst layers were not catalytically active at 450 ${ }^{\circ} \mathrm{C}$.

c) Implementing two successive growths: The highest is the nanocarbon yield, the greater is the augment of electroactive area so an enhanced electrochemical response is expected. In addition, a bigger surface area favours both incorporation and stabilisation of biomacromolecules. The last point is of crucial importance for enzyme-mediated detection mechanisms. With this nanofabrication approach, the reduction of the temperature produces a considerable
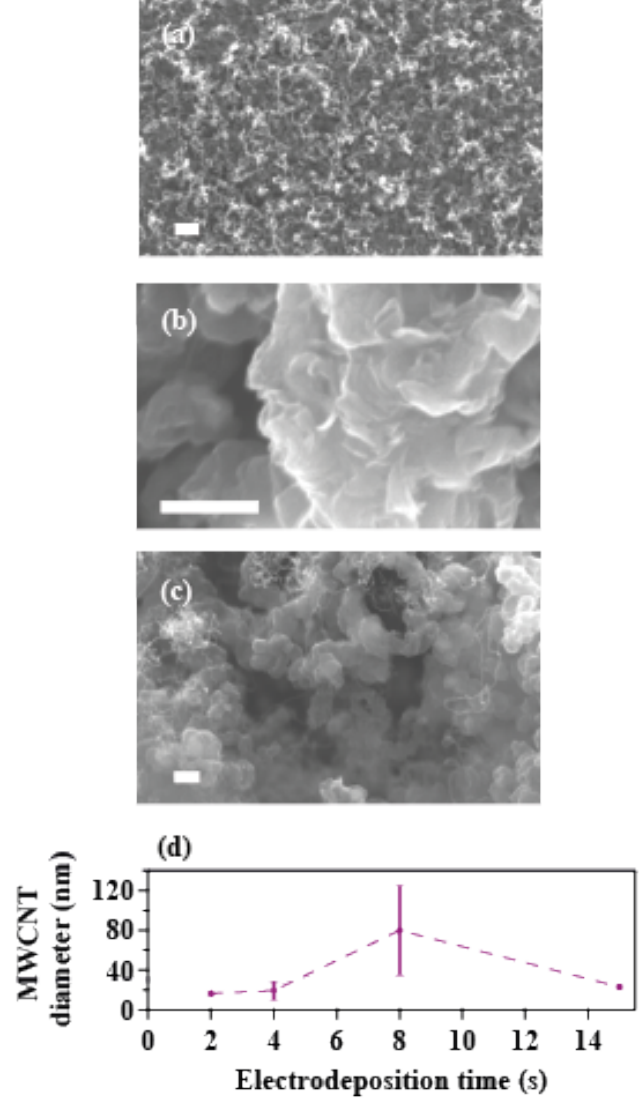

Fig. 6. SEM images of MWCNTs grown on $2 \mathrm{~s}$ (a) of electrodeposited catalyst coating. SEM images of hybrid MWCNTs-nanographite (b) and nanographite (c) produced on $15 \mathrm{~s}$ and $30 \mathrm{~s}$ of deposited $\mathrm{Fe}_{2} \mathrm{Co}$ coatings. Effect of the $\mathrm{Fe}_{2} \mathrm{Co}$ electrodeposition time on the nanotube/nanofiber diameter (e). White bars: $200 \mathrm{~nm}$.

drop in the nanocarbon yield. To increase the yield of the nanostructures, we carried out two successive depositions at $450{ }^{\circ} \mathrm{C}$. After the first synthesis, we deposited thin layers of $\mathrm{Fe}_{2} \mathrm{Co}$ on the top of nanomaterials grown at the first stage (Fig. 8 (a)). After, a second synthesis was carried out keeping identical growth parameters. From 2 $\mathrm{s}$ of electrodeposited catalyst we obtained more carbon nanomaterials, namely CNWs with intercalated MWCNTs (Fig. 8 (b)). Electrodeposition of $\mathrm{Fe}_{2} \mathrm{Co}$ for $4 \mathrm{~s}$ results in an increase of the CNT quantity (Fig. 8 (c)). Also amorphous carbon grows when the catalyst is electrodeposited for a longer interval time.

\section{Application for glucose measurements}

The device with the nanostructured working microelectrodes has been tested for sensing glucose, whose detection was performed by incorporating a glucose oxidase as probe-enzyme onto the electrodes. The morphological change of our nanostructures proves the enzyme incorporation (Fig. 9 (a) and (b)). Glucose oxidase was adsorbed onto the nanomaterials and crosslinked with glutaraldehyde. The sensor was liner up to $1 \mathrm{mM}$ (Fig. 9 (c) and 

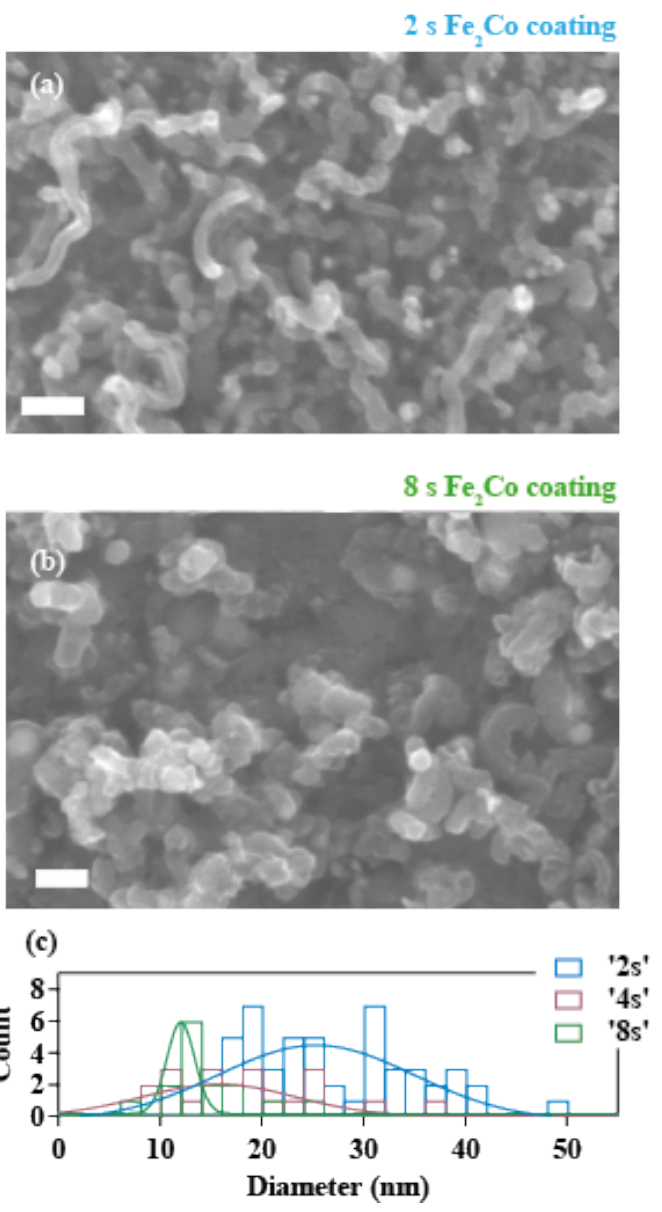

Fig. 7. SEM images of nanomaterials produced at $525^{\circ} \mathrm{C}$ on $2 \mathrm{~s}$ (a) and $8 \mathrm{~s}$ (b) of deposited catalyst layers. (c) Diameter distribution of MWCNTs grown on three different $\mathrm{Fe}_{2} \mathrm{Co}$ coatings at $450{ }^{\circ} \mathrm{C}$. White bars: $100 \mathrm{~nm}$.

(d)). Sensitivity and LOD were $70.4 \pm 0.3 \mu \mathrm{A} /\left(\mathrm{mM} \mathrm{cm}^{2}\right)$ and $5.0 \pm 3.3 \mu \mathrm{M}$, respectively. The enzyme-based sensor shows competitive performance if compared with those reported in literature [15]. Glutaraldehyde was utilised to prolong the time-stability of the enzyme. Sensitivity decreases of about $35 \%$ and LOD did not vary after three days following the protein incorporation. Five days after the enzyme immobilisation, the protein lost its activity toward the glucose detection.

\section{CONClusions}

Carbon nanomaterials are typically synthesised via CVD at $650-1200{ }^{\circ} \mathrm{C}$, temperatures not compatible with a direct CMOS integration. Here, for the first time, we report a method to obtain MWCNTs, nanographite and CNWs down to $450{ }^{\circ} \mathrm{C}$, opening the possibility to a direct integration nanostructures/front-end of CMOS data acquisition circuits. We succeeded in growths on only the selected metal WEs of a multi-panel biosensing-platform avoiding the co-immobilisation of additional binders. Furthermore, we easily obtained different kinds of carbon nanostructures only by changing the nature of the electrodeposited (a)
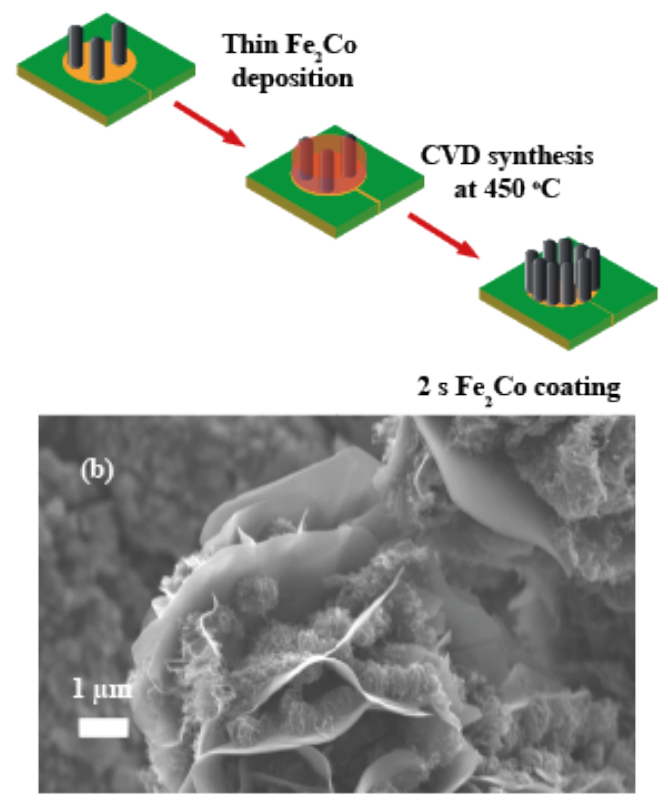

4 s $\mathrm{Fe}_{2}$ Co coating

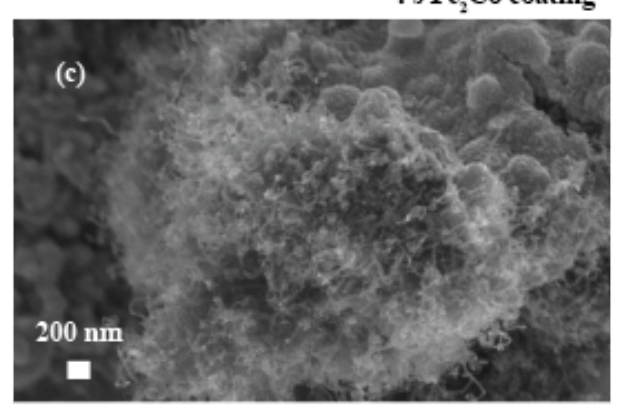

Fig. 8. Two deposition CVD process (a); CNWs and MWCNTs grown onto $2 \mathrm{~s}$ of electrodeposited $\mathrm{Fe}_{2} \mathrm{Co}$ (b); MWCNTs grown onto $4 \mathrm{~s}$ of electrodeposited $\mathrm{Fe}_{2} \mathrm{Co}(\mathrm{c})$.

catalyst thanks to the high versatility of our approach. We succeeded in increasing the nanocarbon yield at 450 ${ }^{\circ} \mathrm{C}$ that is of crucial importance for high performance biodetection. Finally, the described integration method was proven to be robust and highly efficient for oxidasemediated amperometric sensing of glucose.

\section{ACKNOWLEDGMENT}

The authors would like to thank Andrea Cavallini for the design of the microfabricated platform and Laurent Bernard for the preparation of the CVD system. The research was supported by the i-IronIC++ and ERC NanoSys projects. Arnaud Magrez acknowledges financial support from the SCOPES project ${ }^{\circ}{ }^{\circ}$ IZ74Z0 137458 and the European project NAMASEN. 

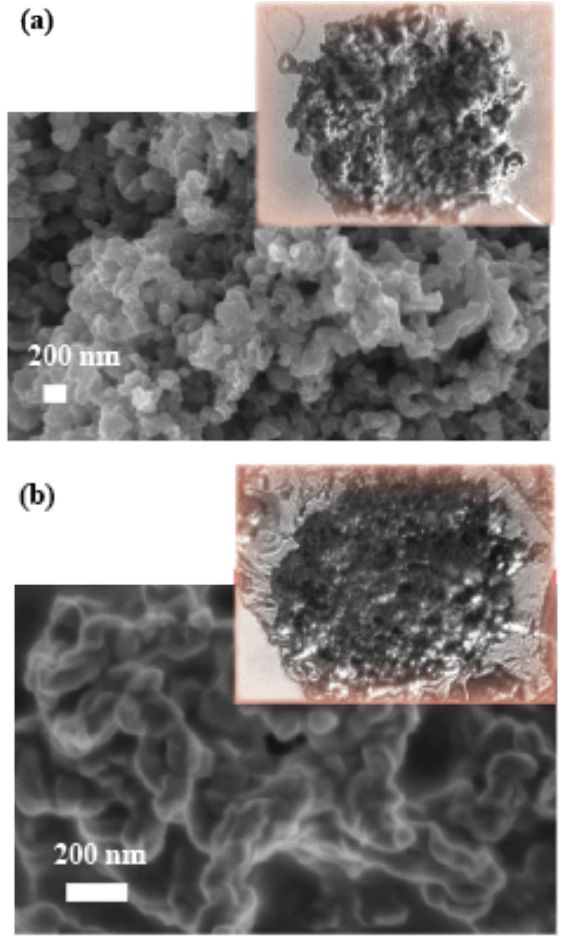

(c)

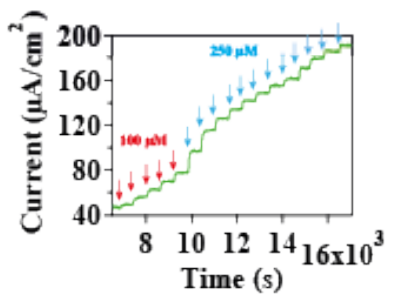

(d)

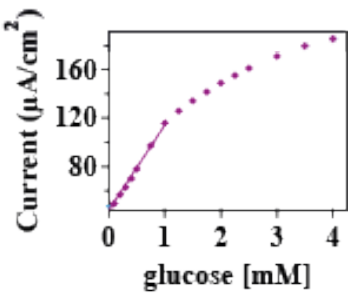

Fig. 9. Optical and SEM images of the nanostructured electrode before (a) and after (b) the protein incorporation. Chronoamperometry (c) and calibration curve (d) of the glucose micro-nanosensor.

\section{REFERENCES}

[1] I. Moser, G. Jobst, and G. A. Urban, "Biosensor arrays for simultaneous measurement of glucose, lactate, glutamate, and glutamine," Biosensors and Bioelectronics, vol. 17, no. 4, pp. 297-302, 2002.

[2] M. Miyashita, N. Ito, S. Ikeda, T. Murayama, K. Oguma, and J. Kimura, "Development of urine glucose meter based on microplaner amperometric biosensor and its clinical application for self-monitoring of urine glucose," Biosensors and Bioelectronics, vol. 24, no. 5, pp. 1336-1340, 2009.

[3] S. Carrara, A. Cavallini, V. Erokhin, and G. De Micheli, "Multipanel drugs detection in human serum for personalized therapy," Biosensors and Bioelectronics, vol. 26, no. 9, pp. 3914-3919, 2011.

[4] S. Carrara, S. Ghoreishizadeh, J. Olivo, I. Taurino, C. Baj-Rossi, A. Cavallini, M. Op de Beeck, C. Dehollain, W. Burleson, F. G. Moussy et al., "Fully integrated biochip platforms for advanced healthcare," Sensors, vol. 12, no. 8, pp. 11013-11060, 2012.

[5] S. Carrara, A. Cavallini, S. Ghoreishizadeh, J. Olivo, and G. De Micheli, "Developing highly-integrated subcutaneous biochips for remote monitoring of human metabolism," in IEEE Sensors conference, 2012, pp. 28-31.
[6] W. Yang, K. R. Ratinac, S. P. Ringer, P. Thordarson, J. J. Gooding, and F. Braet, "Carbon nanomaterials in biosensors: should you use nanotubes or graphene?" Angewandte Chemie International Edition, vol. 49, no. 12, pp. 2114-2138, 2010.

[7] C. T. Chow, M. L. Sin, P. H. Leong, W. J. Li, and K. Pun, "Design and modeling of a cnt-cmos low-power sensor chip," in Nano/Micro Engineered and Molecular Systems, 2007. NEMS'07. 2nd IEEE International Conference on. IEEE, 2007, pp. 12091214.

[8] R. S. Chakraborty, S. Narasimhan, and S. Bhunia, "Hybridization of cmos with cnt-based nano-electromechanical switch for low leakage and robust circuit design," Circuits and Systems I: Regular Papers, IEEE Transactions on, vol. 54, no. 11, pp. 2480-2488, 2007.

[9] Y. Zhou, J. L. Johnson, A. Ural, and H. Xie, "Localized growth of carbon nanotubes on cmos substrate at room temperature using maskless post-cmos processing," Nanotechnology, IEEE Transactions on, vol. 11, no. 1, pp. 16-20, 2012.

[10] J. Wang, M. Musameh, and Y. Lin, "Solubilization of carbon nanotubes by Nafion toward the preparation of amperometric biosensors," Journal of the American Chemical Society, vol. 125, no. 9, pp. 2408-2409, 2003.

[11] I. Taurino, A. Magrez, F. Matteini, L. Forró, G. De Micheli, and S. Carrara, "Direct growth of nanotubes and graphene nanoflowers on electrochemical platinum electrodes," Nanoscale, vol. 5, no. 24, pp. 12 448-12455, 2013.

[12] V. T. Renard, M. Jublot, P. Gergaud, P. Cherns, D. Rouchon, A. Chabli, and V. Jousseaume, "Catalyst preparation for CMOScompatible silicon nanowire synthesis," Nature nanotechnology, vol. 4, no. 10, pp. 654-657, 2009.

[13] J. Shen and C.-C. Liu, "Development of a screen-printed cholesterol biosensor: Comparing the performance of gold and platinum as the working electrode material and fabrication using a selfassembly approach," Sensors and Actuators B: Chemical, vol. 120, no. 2, pp. 417-425, 2007.

[14] M. Pumera, T. Sasaki, and H. Iwai, "Relationship between carbon nanotube structure and electrochemical behavior: heterogeneous electron transfer at electrochemically activated carbon nanotubes," Chemistry-An Asian Journal, vol. 3, no. 12, pp. 2046-2055, 2008.

[15] Y. Chen, J. Huang, and C. Chuang, "Glucose biosensor based on multiwalled carbon nanotubes grown directly on Si," Carbon, vol. 47, no. 13, pp. 3106-3112, 2009.

[16] W. Rasband, "ImageJ," U. S. National Institutes of Health, Bethesda, Maryland, USA, 1997-2011, http://imagej.nih.gov/ij/.

[17] A. Magrez, J. Seo, R. Smajda, B. Korbely, J. Andresen, M. Mionić, S. Casimirius, and L. Forró, "Low-temperature, highly efficient growth of carbon nanotubes on functional materials by an oxidative dehydrogenation reaction," ACS Nano, vol. 4, no. 7, pp. 37023708, 2010. 